\title{
The use of 3D printing for osteopathic medical education of rib disorders
}

https://doi.org/10.1515/jom-2020-0020

Received February 9, 2020; accepted October 14, 2020; published online February 11, 2021

\begin{abstract}
Context: With the advent of increasingly accessible threedimensional (3D) printing, the possibility to efficiently design and generate prototype innovations is also increasing. This type of manufacturing can potentially enhance medical education by allowing design of models specific to osteopathic manipulative medicine (OMM).

objectives: To determine the viability of a 3D-printed mechanically moveable rib cage in enhancing the teaching of rib osteopathic principles.
\end{abstract}

Methods: A single-blind, qualitative study was conducted to evaluate the use of educating students with this novel 3D-printed, movable rib model vs. a traditional static rib model. A total of 237 first-year medical students participated in the study and received the same standardized lecture on the rib dysfunction. Students were also assigned at random to either a comparison group, which would utilize the 3D printed rib model, or the control group, which would utilize the traditional static model. Students would also complete an entrance and exit surveys assessing subjective scores of overall student satisfaction and objective scores for knowledge of OMM rib dysfunction and treatment. An independent samples t-test was applied to assess potential differences between select student evaluation scores (those with continuous variables) of the rib model in the comparison and experiment groups. Chisquare goodness of fit test was conducted to determine if there were any significant differences in entry and exit

*Corresponding author: Kevin Moriles, MS, Dr. Kiran C. Patel Nova Southeastern University College of Osteopathic Medicine, 3301 College Avenue S University Drive, Fort Lauderdale, FL 33314-7796, USA, E-mail: km2397@mynsu.nova.edu

Amanda Ramnot, BS, Michael Lai, BS, Robin J. Jacobs, PhD, MSW, MS, MPH and Yasmin Qureshi, EdD, DPT, MHS, Dr. Kiran C. Patel College of Osteopathic Medicine, Nova Southeastern University, Fort Lauderdale, FL, USA survey responses between the two groups. Descriptive statistics of the mean and standard deviation were also reported.

Results: For both comparison and control groups, the mean score on an 11-point scale for the evaluation question, "Please rank on a scale of 0-10 how helpful you thought the rib models were to your education," was 9.08 (SD, 1.397). Independent t-test results showed that the comparison group had higher scores than the control group when queried about whether they felt the model accurately depicted the material presented (comparison group mean, 9.55 [SD, 978] vs. control group mean, 9.06 [SD, 1.33; $t(235)=3.253 ; \mathrm{p}=0.01)$. Chi-square test of goodness-of-fit showed that the differences between the number of correct answers chosen by participants for Item 3 (a case-based question asking students which rib they would treat for a patient presenting to an OMT clinic) was statistically significantly higher for the comparison group (51.9\% correct in comparison group vs. $48.1 \%$ in control group), even though both groups scored similarly on this item during the entry survey.

Conclusions: The results of this study suggest that utilizing 3D printing to demonstrate somatic dysfunctions of the rib cage may improve understanding and student satisfaction for diagnosis and treatment.

Keywords: 3D printing; medical education; OMM; OMT; rib disorder; somatic dysfunction.

Three-dimensional (3D) printing, also referred to as additive manufacturing or rapid prototyping printing, is a method in which 3D objects are created by the automated layering of materials such as plastic. The $3 \mathrm{D}$ printer is instructed by a user-input computer-aided design (CAD) file to build the object layer by layer. The printer head moves along the $x-y$ plane, layering material along the $z$-axis vertically to completion [1]. With the inexpensive nature of materials, 3D printing allows for rapid and costeffective prototyping of models. Also, the use of 3D printing in education allows production of multiple identical prototypes that can be scaled to achieve the size desired [2]. 
As it pertains to the medical field, 3D printing has been shown to be an inexpensive and rapid solution for educational purposes, the visualization of previous 2D imaging, and in planning surgical procedures [2-5]. In one study [6], a method to print patient-specific portions of the skull was developed, costing as little as $\$ 1-\$ 5$ US per model and taking less than 14 hours to complete [6].

Likewise, 3D printing can provide an alternate method for planning surgeries and aid in the teaching of medical students and residents [3, 7]. In a previous study [7], patient-specific 3D kidney models were found to improve the understanding of renal anatomy in patients with renal tumors for both urologists and medical students, suggesting that this method can be utilized in perioperative planning as well as in education of medical students.

An additional study [8] comparing the effectiveness of 3D printed models vs. cadaveric material used to teach external cardiac anatomy showed that posttest scores were significantly higher for the group educated with 3D prints vs. cadaveric materials [8]. Other authors [9] conducted a systematic review and metaanalysis on the clinical value of $3 \mathrm{D}$ printed models, concluding that $3 \mathrm{D}$ printed heart models could be used in the field of medical education to improve learning experience and satisfaction. The review also found that 3D printed heart models played a role in improving the teaching and understanding of complex congenital heart diseases vs. simple congenital heart diseases [9]. This concept was further supported by an unrelated study [10] in which pediatric residents being taught Tetralogy of Fallot with 3D models gave higher composite learner satisfaction scores than residents taught with 2D images [10]. Results in another randomized, controlled trial [11] showed that the use of 3D printed models for medical students was associated with improved learning of bone spatial anatomy and fractures in particular complex anatomical locations when compared to 2D radiographic images. Students instructed using 3D models not only scored higher on tests, but also took significantly less time to answer questions when compared to counterparts who received $2 \mathrm{D}$ instruction [11].

Osteopathic physicians should be aware of these studies because a thorough understanding of bony anatomy is required for comprehension of specific Osteopathic Manipulative Techniques (OMT). In this field, 3D printing has great potential to produce cost-effective tools that enhance understanding of osteopathic principles and practice, which could enhance both medical education and the education of patients undergoing osteopathic treatments. For example, a previous randomized clinical trial [12] investigated the efficacy of using 3D printed models in the treatment of fractures, concluding that their use helped physicians plan and perform the operation, while also providing a method for more effective communication between doctor and patient [12].

Despite this, there are currently no osteopathicspecific models available for patient or student education on somatic dysfunctions. A review of the literature also revealed no current studies evaluating the use of $3 \mathrm{D}$ printing in teaching osteopathic-specific education. Therefore, we designed this study to explore the possible benefits of using a 3D printed rib model to teach osteopathic medical students the principles of rib dysfunction and treatment.

The choice of modeling rib dysfunction and treatment was multifactorial. The ribs move in synchrony with the breathing cycle; however, because of the various bony and cartilaginous attachments of the thoracic cage, ribs can exhibit somatic dysfunction through various classifications in line with their predominant method of articulation. Ribs classified as exhibiting mostly "pump-handle" motion articulate anteriorly and posteriorly through costovertebral and costotransverse junctions. Ribs classified as "bucket-handle" predominantly exhibit motion through the costotransverse and the costochondral articulations laterally. Finally, ribs defined as "caliper" predominantly increase the transverse diameter of the thoracic cage with inhalation. Although the ribs can display predominant motions in line with these classifications, it is important to note that all ribs are capable of exhibiting any of these motions. Somatic dysfunction of the rib is a dysfunction in which the position of movement of a rib is altered. These dysfunctions can be divided into two groups: inhalation dysfunction or exhalation dysfunction. In an inhalation dysfunction, the rib of interest will move freely in the cephalad direction during inhalation but will not move caudally during exhalation. Alternatively, in an exhalation dysfunction, the rib of interest will move freely in the caudad direction during exhalation but will not move cephalically during inhalation. Also, contiguous sets of ribs can have "group" dysfunctions in which the positioning or movement of two or more ribs can be altered. In the instance of a group dysfunction, there is generally a "key" rib that is responsible for the dysfunction and it is then the target of treatment. In an inhalation dysfunction, the key rib is the lowest in the group of affected ribs; in an exhalation dysfunction, the key rib is the uppermost rib in the group dysfunction [13]. When managing a rib dysfunction, multiple treatment approaches can be utilized, including counterstrain, muscle energy, and high-velocity low-amplitude (HVLA) techniques. To better understand somatic dysfunctions, it can be helpful to first have an understanding of the anatomy of the structures 
involved [14] - in the case of this study, it was the multiple points of attachments of ribs, their articulations, and their predominant motions. A thorough understanding of 3D anatomic relationships is essential when making physical diagnoses. Having a tangible 3D model that clearly demonstrates the various motions of the ribs can confer spatial understanding when visualizing and treating such dysfunctions.

The purpose of this study was to demonstrate somatic dysfunction of the rib cage through a novel prototype design that displays rib biomechanics. The design of the novel 3D model in the current study allowed for $360^{\circ}$ articulation of each rib, bound only posteriorly by a vertebral attachment such that the dynamic and spatial relationships could be better appreciated. Through entry and exit surveys we compared knowledge and subjective program satisfaction for learning conducted with the prototype 3D printed rib model vs. a traditional static model that did not allow for replication of rib movement.

\section{Methods}

The Nova Southeastern University Institutional Review Board determined that this study exempt under 45 CFR 46.101(b). The project adhered to policies outlined in the Family Educational Rights and Privacy Act (FERPA) (20 U.S.C. § 1232g; 34 CFR Part 99) regarding the protection of students' educational records and privacy.

The prototype rib model was developed between October 2018 and February 2019. Once the prototype design was finalized, the printing of individual parts and assembly of complete models were completed in February and March 2019. The study took place on March 21st, 2019, over two separate lab sessions that lasted approximately 2 hours each. Data analysis initially began in April 2019 and was completed in August 2020.

Participants were first-year osteopathic medical students enrolled at a large osteopathic medical school in Florida. Two hundred and thirty-seven of 257 students enrolled in the class participated in the study. All students who participated in the study received a letter explaining the research and requesting informed consent. As this was a single-blind qualitative study, participants were assigned at random to a comparison group or control group designation within the osteopathic laboratory class. The participants were placed on a deidentified Excel spreadsheet (Microsoft, Inc.) and assigned a comparison group or control group designation with the RAND (random) function on excel. Students from the comparison group participated in the first laboratory session and received instruction with the novel 3D rib model; students from the control group participated in the second laboratory session and received instruction with the traditional model.

\section{D printed rib model and traditional rib model}

The prototype 3D rib model was designed to emulate rib biomechanics in relation to the vertebra and various rib somatic dysfunctions

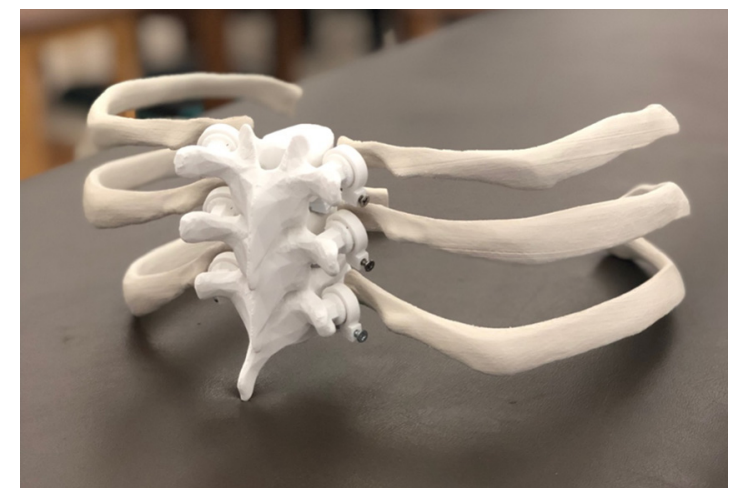

Figure 1: Posterolateral view of the assembled three-dimensional prototype rib model.

(Figure 1). Each model was designed with three thoracic vertebra levels with a respective rib on each side, for a total of six ribs. The prototype model was built from Acrylonitrile Butadiene Styrene (ABS) plastic material [15] (Figure 2). The individual materials utilized in assembly included individual printed ribs and vertebrae, screws, plastic rings, and foam sheets to serve as intervertebral disks. The balljoint for articulation was adapted from Gerefalk's "Locking Ball and Socket Arm" and licensed under Creative Commons CC BY-SA 3.0 for adaptation [16]. The rib and vertebra were adapted by Mitsuhashi's "BodyParts3D," and licensed under CC Attribution-Share Alike 2.1 Japan [17]. Files were exported out of .obj format and reformatted for

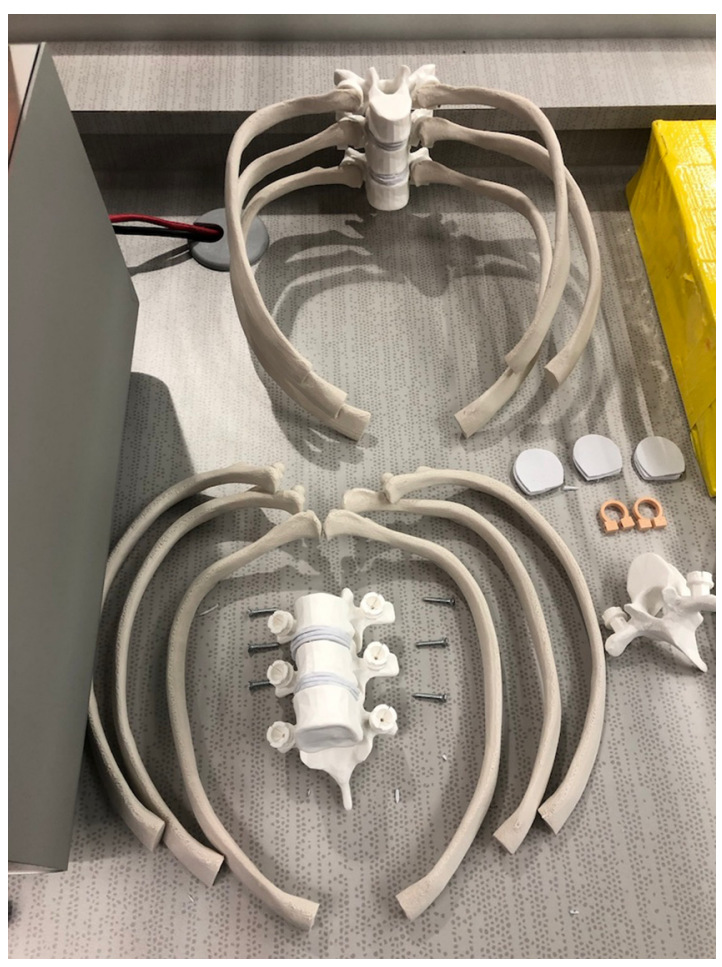

Figure 2: Superior and anterior view of an assembled threedimensional prototype rib model and the individual materials used in assembly: individual ribs and vertebrae, screws, plastic rings, and foam sheets cut in the shape of the vertebral body of the vertebrae. 


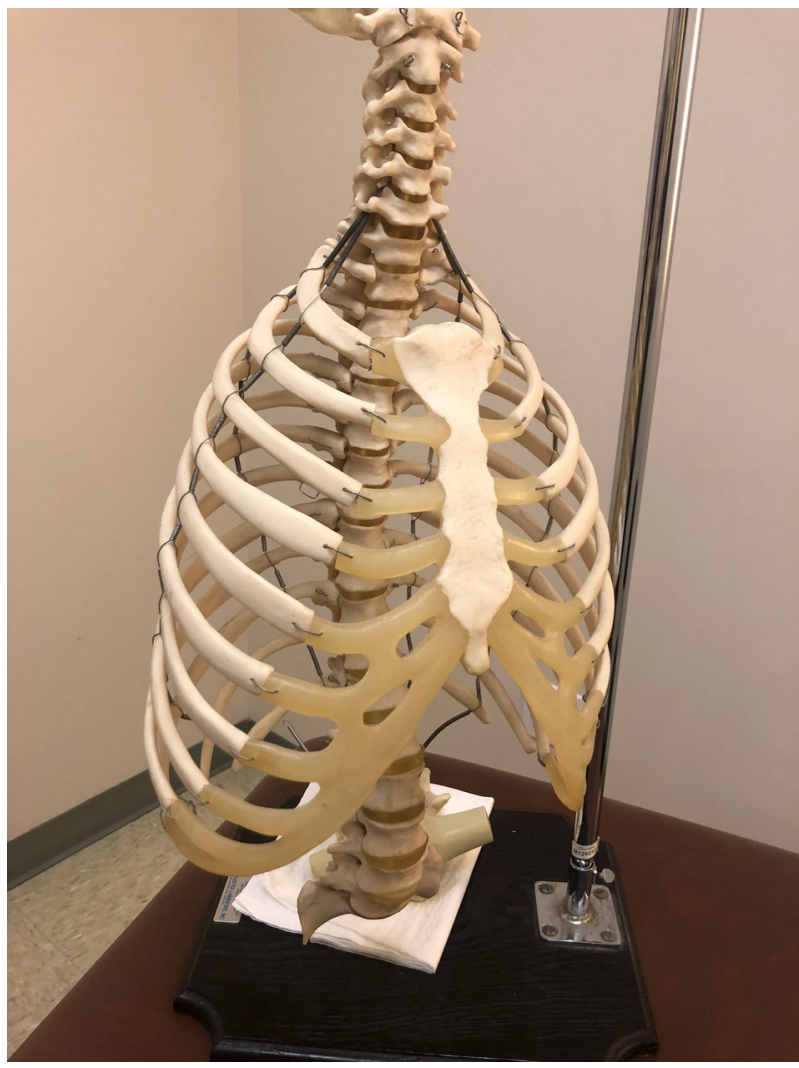

Figure 3: Anterior view of the traditional model.

Meshmixer software (Autodesk, Inc). Within the Meshmixer program, the joint was placed at the superior costal facets on both sides of the vertebra. The ball portion was placed on the articular rib tubercle to allow for attachment to the receptive joint on the vertebra to form the articulation of the completed rib model. The ball and socket were locked with an equally 3D printed plastic ring that wrapped around the joint. A screw was used to lock this plastic ring in place and tighten around the joint for continued stabilization. The prints were scaled up by $10 \%$ and printed at default balanced settings with $10 \%$ infill \& supports. Because of the novel joint design, both pump handle and bucket handle rib motions could be accurately displayed. In relation to the joint, the rib could move within a 90-degree arch in the transverse plane and a $180^{\circ}$ arch in the sagittal plane. This allowed the model to display the elevation and forward motion of the ribs in pump handle motion. The model also had the ability to raise and lower the middle of the rib, representing bucket handle movement (Figure 3).

The traditional model (Figure 4) was not designed to emulate physiological rib movement or rib somatic dysfunction. The traditional rib model did not have the novel ball and socket, nor the locking plastic ring design. The traditional rib model was also not produced with Meshmixer software nor through 3D printing. This model had the same dimensions of vertebrae and ribs of the 3D prototype rib model, and it also included a sternum and manubrium like that of a traditional plastic skeletal model. The traditional model also included thoracic ribs 1-12 but lacked rib biomechanics.

Paper surveys were delivered to each individual student and collected by three authors (K.M., A.R., M.L.). On study enrollment, participants completed an entry survey consisting of three questions

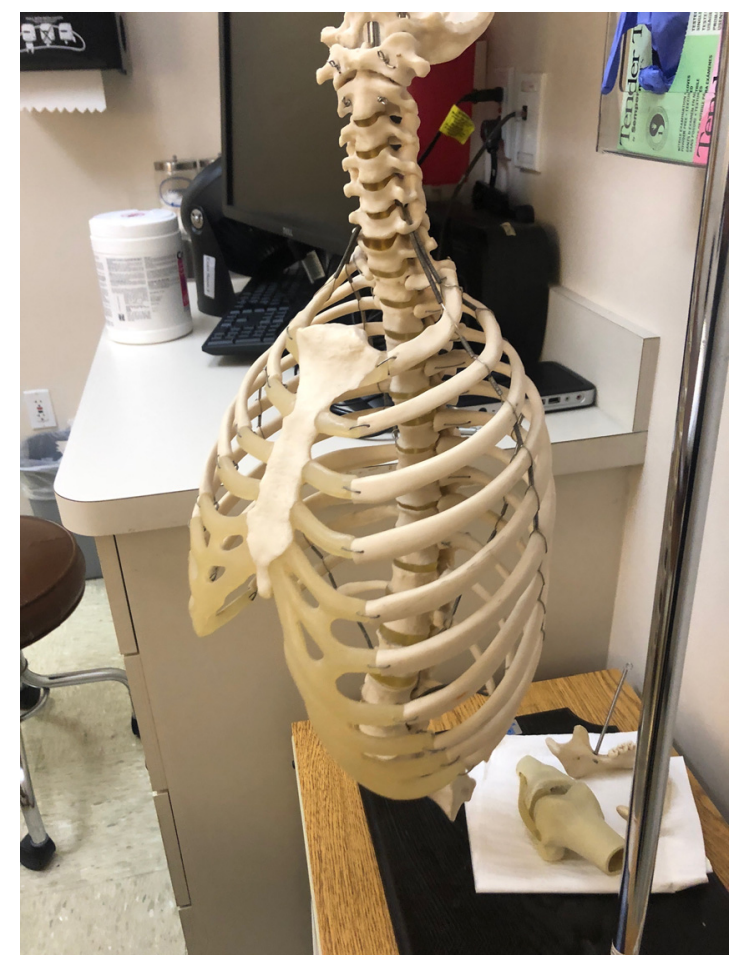

Figure 4: Lateral view of the traditional model.

about their knowledge of OMTs regarding rib diagnosis and treatment (Figure 5). Each question had a single correct answer out of four possible answers. Each item was scored separately as incorrect or correct (dichotomous variable). Possible total scores ranged from 3-9, with higher scores indicating more questions answered correctly.

After completion of the entry survey, each group participated in a standardized OMT lecture during a laboratory session where they were taught how to diagnose and treat rib dysfunctions with their group's respective rib model. The lecture consisted of three, 10- to 15-minute sessions by the main instructor situated in the center of the room; mounted computer monitors throughout the classroom displayed the instructor, enabling all students to have highly visible instruction. The main instructor was the Chairman of the Department of Osteopathic Principles and Practices, who was not an author on this paper. During the first session, the instructor demonstrated and explained the various movements of the ribs using the selected model; during the second session, the instructor demonstrated inhaled somatic dysfunctions; during the third session, the instructor demonstrated exhaled somatic dysfunctions. The instructor also demonstrated how to treat a given dysfunction with the muscle energy technique on a student volunteer during the second and third sessions.

Each session was followed by an additional 10-15-minute session with smaller groups of five to seven students. These were supervised by a physician, physical therapist, or predoctoral osteopathic practices and principles fellow. During the smaller group sessions, each student was able to use their respective model to visualize the pumphandle and bucket-handle motions. Also, students were able to visualize inhaled and exhaled somatic dysfunctions. The amount of time spent for the lesson, educational content, and main class instructor were standardized (constant) across each group (Figure 6). At the end of the laboratory session, participants completed an exit 
1. A patient presents to the $\mathrm{OMM}$ clinic with rib pain. You diagnose a rib 8 inhaled dysfunction (bucket handle predominant) on the left side. Which of the following is true?

a. At midaxillary line, rib 8 will be closer to rib 7 in an inhaled dysfunction at rib 8 .

b. At midaxillary line, rib 8 will be closer to rib 9 in an inhaled dysfunction at rib 8 .

c. There will be no change regarding rib distances from each other.

d. The information provided is insufficient to determine where rib 8 will be.

2. A patient presents to the $\mathrm{OMM}$ clinic complaining of rib pain. You diagnose an exhaled dysfunction at rib 7-8 (bucket handle predominant). Which of the following statements describes the movement of the rib during a normal breathing pattern?

a. Shaft moves upward on inhalation; does not move caudally on exhalation.

b. Shaft moves upward on exhalation; does not move cephalically on inhalation.

c. Shaft moves downward on exhalation; does not move cephalically during inhalation.

d. Shaft moves downward on inhalation; does not move caudally during exhalation.

3. A patient presents to the OMT clinic with pain while breathing. You evaluate the patient and while palpating at the costotransverse junction they feel a deep aching pain on the right. At the sternum, you notice that ribs 6,7 , and 8 are all closer together, while the spaces between ribs 8 and 9 are widened. Ribs $6-8$ move freely cephalad but are restricted in caudad motion. Which rib do you treat?
a. Rib 6
b. Rib 7
c. Rib 8
d. Rib 9

Figure 5: Entry and exit survey questions regarding rib knowledge. OMM, osteopathic manipulative medicine; OMT, osteopathic manipulative treatment. survey that evaluated knowledge learned, select demographics (age, sex, and the highest level of completed education before entering medical school), and subjective evaluations of rib models.

The exit survey (Figure 6) included the same three items in the entry survey, along with seven additional items assessing student perceptions of the models. Six of these additional items used a visual analog scale (0-10) for responses, with another using a dichotomous response set (yes/no) to assess whether the participant felt the use of models enhanced their understanding of rib anatomy, dysfunction, and treatment (Figure 7). The final item assessed the participants' perceived minimum amount of necessary exposure time to the new rib model to facilitate learning rib somatic dysfunctions.

\section{Preliminary data analysis}

Data from assessments were input into a separate Excel spreadsheet from group assignments. Data was then coded, and each researcher completed a data check of every survey to ensure responses were consistent with the questions asked. A code check was completed three times to ensure each survey was also properly coded into the Excel spreadsheet. A range and constraint check were also completed to ensure survey inputs were within a minimum/maximum range of values to further ensure data validation. The data was then imported into Statistical Package for the Social Sciences (SPSSv.26) software.

We predetermined that cases with more than 33\% missing data would be deleted from the analysis, but no cases were removed as none fit the requirement for deletion. This resulted in 123 participants in the control group and 114 participants in the comparison group.

An independent samples t-test was used to assess potential differences between select student evaluation scores (those with continuous variables) of the rib model in the comparison and experiment groups. Chi-square goodness of fit test was conducted to determine if there were any significant differences in the responses between the two groups' entry and exit surveys. Descriptive statistics (mean, standard deviation) are reported.

\section{Results}

Two-hundred and fifty-seven students were eligible for the study and 237 participated (92\%).

Thirteen participants (5.5\%) were age 21 years or younger, $185(78.1 \%)$ participants were age 22-26, $35(14.8 \%)$ were age $27-31$, three $(1.3 \%)$ were age $32-36$, and one $(0.4 \%)$ was age $36-40$. One hundred and eleven (46.8\%) participants were men and 126 (53.2\%) participants were women. One hundred and seventy-nine (75.5\%) participants held a Bachelor's degree, 57 (24.1\%) held both a Bachelor's and Master's, and one (0.4\%) held other degrees. We did not identify any statistical relationships between the characteristic demographics of the sample group and the results of the study.

In both groups, 235 (99.2\%) students agreed that the use of models enhances their understanding of rib anatomy, dysfunction, and treatment. Results also showed that students in both groups were interested in learning how to diagnose and treat rib somatic dysfunctions with OMT (mean value on analog 11-point scale, 7.52; standard deviation [SD], 2.403). Students in both groups felt their model accurately depicted the material presented (mean value on analog 11-point scale, 9.3; SD, 1.196). Students in both groups felt rib models were helpful in their education (mean value on analog 11-point scale, 9.08; SD, 1.397).

The independent t-test results showed that the comparison group $(n=114)$ had higher scores (mean, 9.55 [SD, 0.978]) when queried whether they felt the model accurately depicted the material presented when compared 
Post-Lab Rib Questions/Demographics

1. Age
a) 21 or younger
b) 22 to 26
c) 27 to 31
d) 32 to 36
e) 36 to 40
f) 41 or older

2. $\operatorname{Sex}$

a) Male

b) Female

3. Highest level of completed education (before medical school)

a) Bachelor's

b) Master's

c) Doctorate

d) Other

\section{OPP Model Evaluation}

1. Do you feel that the model accurately depicted the material presented? (please circle a number)

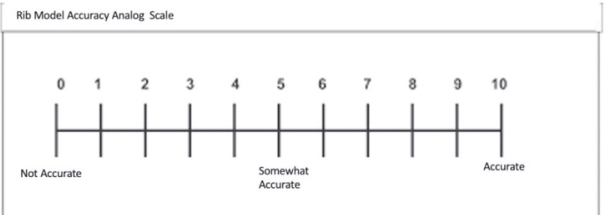

2. How interested are you in learning how to diagnose and treat rib somatic dysfunctions with OMT? (please circle a number)

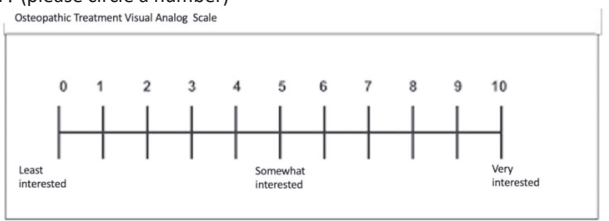

3. Do you feel that the use of models enhances your understanding of rib anatomy, dysfunction, and treatment?

$$
\text { a. yes }
$$

4. Please rank on a scale of 1-10 how helpful you thought the rib models were to your education.

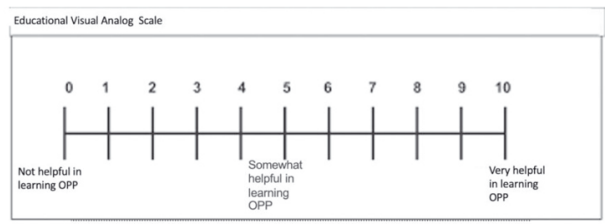

\section{Evaluation of Knowledge}

1. How would you characterize your performance in OPP in the past? (expectations met?)

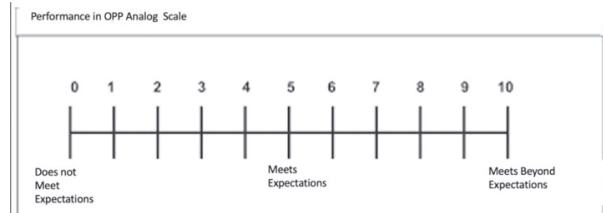

2. How comfortable do you feel with the anatomy and somatic dysfunctions of ribs?

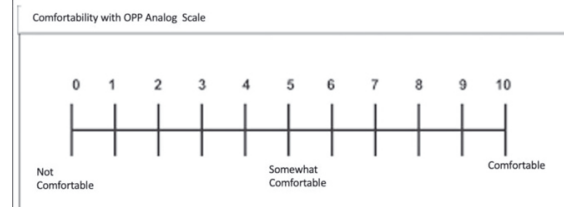

Figure 6: Exit survey questions assessing participant demographics and evaluation of the 3D model. OMT, osteopathic manipulative therapy; OPP, osteopathic principles and practice. 

What is the minimum amount of time that exposure to this rib model would be necessary to facilitate learning rib somatic dysfunctions?
a) 15 minutes
b) 30 minutes
c) 60 minutes or more
d) I do not feel that this rib model would be beneficial in learning about rib somatic dysfunction.

Figure 7: Exit survey question assessing perceived learning time.
Table 1: Comparison of responses to case-based knowledge questions ${ }^{a}$ on the entry and exit survey.

\begin{tabular}{lrr}
\hline $\begin{array}{l}\text { Survey } \\
\text { item }\end{array}$ & $\begin{array}{r}\text { Correct answers, entry } \\
\text { survey }\end{array}$ & $\begin{array}{r}\text { Correct answers, exit } \\
\text { survey }\end{array}$ \\
\hline Item 1 & $166(72.2 \%)$ & $199(84.0 \%)$ \\
Item 2 & $141(61.1 \%)$ & $185(78.1 \%)$ \\
Item 3 & $84(36.5 \%)$ & $174(73.4 \%)$ \\
\hline
\end{tabular}

${ }^{a}$ Questions shown in Figure 5.

with the control group (mean, 9.06 [SD, 1.33]; $t(235)=3.253$; $\mathrm{p}=0.01$ ).

The chi-square test of goodness-of-fit showed that the differences between the correct answers chosen by participants in the two groups for Item 3 (a case-based question asking students which rib they would treat for a patient presenting to an OMT clinic) was statistically significant, even though both groups scored similarly on this item during the entry survey $\left(\chi^{2}[1, \mathrm{n}=237]=5.97 ; \mathrm{p}=0.015\right.$; $51.9 \%$ correct in comparison group vs. $48.1 \%$ in control group).

Table 1 shows summary statistics for the entry and exit survey items.

A post-hoc analysis was conducted to assess the power to detect a medium effect size between the experimental group ( $\mathrm{n}=114)$ and the control group $(\mathrm{n}=123)$ in resulting knowledge scores. Our study ( $\mathrm{n}=237,0.05$ alpha, 2-tailed test), had power in excess of 0.969 given a population correlation of medium effect size $(d=0.50)$, power of 0.86 given a population correlation of 0.40 , power of 0.63 given a population correlation of 0.30 , power of 0.33 given a population correlation of 0.20 , and power of 0.12 given a population correlation of 0.10 . For the chi-square goodness of fit test, a post-hoc analysis was conducted to assess the power to determine if there were any significant differences in the responses between the experimental and control groups' entry and exit surveys. For this test, our study had power in excess of 0.964 given a population correlation of medium effect size $(d=0.30)$. Thus, our study was well-suited for detecting relatively large population effects - although perhaps not well-designed for finding (or establishing the existence of) smaller effects [18].

\section{Discussion}

On survey analysis, a very high percentage of students agreed that using any model enhanced their understanding of rib anatomy, dysfunction, and treatment. Further, students in this study generally agreed that the use of models, whether 3D printed or not, could affect education on rib anatomy, somatic dysfunction, and treatment. This is possibly due to the ability of models to help visualize the motion and position of each rib in relation to a particular rib somatic dysfunction diagnosis. Statistical analysis revealed that a significant number of students did see some benefit from the use of models. This conclusion aligns with the use of other 3D printed models in the literature, such as a study that compared the effectiveness of 3D printed models vs. cadaveric material and found significantly higher post-test scores in the 3D prints group compared to the cadaveric materials [8]. Thus, the 3D printed model prototyped for this experiment was designed to emulate rib dysfunctions that would otherwise be difficult to visualize. A limitation of the prototyped design was that the model was unable to emulate caliper rib motion seen in ribs 11 and 12. While the three vertebra and respective ribs were able to emulate pump and bucket handle motions seen in ribs $1-10$, the caliper rib motions would require a smaller set of ribs and a different design in order to emulate that unique motion. A future design would create a completed thoracic vertebra that would include ribs 1-12 to emulate all three types of rib motions.

An independent samples t-test of the study revealed that the comparison group had higher scores in feeling the model accurately depicted the material presented than those in the control group. This finding suggests that the 3D printed prototype was successful in emulating somatic rib dysfunction in comparison with a traditional static rib model.

The chi-square of goodness-of-fit also revealed that a statistically significant number of students in the comparison group chose the correct answer on the exit survey about a question requiring spatial understanding more than students in the control group. Both groups scored similarly on this item during the entry survey. This finding 
shows that the 3D model may improve a student's understanding through the enhancement of spatial understanding. It is important to note that more research is needed to determine the viability of 3D printing in clinical education. Direct causation could not be determined in this study, as there are other variables to consider in this finding. It is possible that using models in general, regardless of the model type, could contribute to this increase in understanding. Also, both groups were instructed on the diagnosis and treatment of somatic rib dysfunction after the entry survey, which may also correlate to the improvement of understanding. This study is preliminary and serves as an introduction to the use of 3D printing in osteopathic medicine. Additional research should focus on other osteopathic concepts such as sacral and vertebral dysfunction, in addition to rib dysfunction, to fully evaluate the use of 3D models in osteopathic medicine.

While this study was very limited in its ability to support the enhancement of osteopathic education, the study does serve as a proof of concept. 3D printing offers an inexpensive production of any prototype design. ABS is a hard plastic that can create a product that is both durable and inexpensive [19]. Materials like ABS are typically utilized to produce sporting equipment, protective headgear, and bumpers for the automotive industry [19]. A review of cost revealed that $145.3 \mathrm{~g}$ of the plastic filament were required to complete a single rib model. Each gram of ABS costs 0.022 cents, resulting in each model costing only $\$ 3.20$ to produce. Additional models produced via 3D printing will likely cost a similar amount. This contrasts with other forms of manufacturing, where full rib models can cost up to $\$ 402$ [20]. As such, 3D printing offers a costeffective solution in designing models for osteopathic medicine. Osteopathic physicians should be aware of these findings due to the ability for 3D printing to serve as an alternative method of producing highly specific models to demonstrate osteopathic concepts. Further research could strengthen data on the use of such 3D printed models targeting different OMTs. Examples of future trials of 3D printed models could include prototype sacrum and pelvis models; these could likewise be tested with surveys and statistical analysis.

\section{Conclusions}

The results of the study suggest that 3D printed models may serve as useful and effective educational adjuncts to demonstrate somatic dysfunctions of the rib cage during teaching of osteopathic principles because they enable spatial visualization, thus potentially improving student understanding and satisfaction in the diagnosis and treatment of rib somatic dysfunction.

In addition to showing increased perceived student satisfaction through the use of these models, our results showed that students educated with our 3D prototype model also more often correctly answered questions about rib mechanics than their counterparts educated with a traditional model, suggesting that the use of 3D models aided in student understanding. Considering at the minimal cost of only $\$ 3.20$ to produce each model, 3D printing may offer a more financially appealing alternative compared with other traditional static anatomic models. Future research should focus on designing additional models on other osteopathic concepts to enforce this novel adjunct to teaching.

Acknowledgements: The authors thank Dr. David R. Boesler and the Department of Osteopathic Principles and Practice (OPP) for allowing us to work with the first-year medical students at Nova Southeastern University. We thank him for also serving as the main instructor in this course. We also thank faculty members Dr. Patrick Barry, Dr. Roody Joseph, Dr. Rohit Mehra, Dr. Nathan Widboom, and Dr. Alessandra Posey for serving as supervising faculty. We thank the Predoctoral OPP Fellows Mr. Michael Goldstein, Ms. Robin Mata, Ms. Gabrielle McDermott, Ms. Sara Rodriguez, Ms. Gabriela Teixeira, and Mr. Nicholas Wawrzyniak for their help in both supervising and instructing the students. We thank Mr. Tariq Rahaman for his assistance in producing the model used for the experiment, and we are grateful for his help optimizing the 3D printing device to ensure smooth production. Last, we would like to thank Mr. Christopher Samaan for his input on methods to create movable joints on the model. We appreciate his help and all those acknowledged in making this project possible.

Research funding: None reported.

Author contributions: Mr. Moriles, Ms. Ramnot, and Mr. Lai provided substantial contributions to conception and design, acquisition of data, or analysis and interpretation of data; Dr. Qureshi and Dr. Jacobs drafted the article or revised it critically for important intellectual content; all authors gave final approval of the version of the article to be published; and all authors agree to be accountable for all aspects of the work in ensuring that questions related to the accuracy or integrity of any part of the work are appropriately investigated and resolved.

Competing interests: Authors state no conflict of interest. 
Informed consent: Informed consent was obtained from all individuals included in this study.

Ethical approval: The Nova Southeastern University Institutional Review Board determined this study exempt under 45 CFR 46.101(b).

\section{References}

1. Ursan ID, Chiu L, Pierce A. Three-dimensional drug printing: a structured review. J Am Pharm Assoc (2003) 2013;53:136-44.

2. Mason J, Visintini S, Quay T. An overview of clinical applications of 3-D printing and bioprinting. Ncbi.nlm.nih.gov. Published 2020. Available from: https://www.ncbi.nlm.nih.gov/books/ NBK542711/ [Accessed 1 Aug 2020].

3. Mardis NJ. Emerging technology and applications of 3D printing in the medical field. Mo Med 2018;115:368-73.

4. McMenamin PG, Quayle MR, McHenry CR, Adams JW. The production of anatomical teaching resources using threedimensional (3D) printing technology. Anat Sci Educ 2014;7: 479-86.

5. Gardin C, Ferroni L, Latremouille C, Chachques JC, Mitrečić D, Zavan B. Recent applications of three-dimensional printing in cardiovascular medicine. Cells 2020;9:742.

6. Naftulin JS, Kimchi EY, Cash SS. Streamlined, inexpensive 3D printing of the brain and skull. PloS One 2015;10:e0136198.

7. Lee H, Nguyen NH, Hwang SI, Lee HJ, Hong SK, Byun SS. Personalized 3D kidney model produced by rapid prototyping method and its usefulness in clinical applications. Int Braz J Urol 2018;44:952-7.

8. Lim KH, Loo ZY, Goldie SJ, Adams JW, McMenamin PG. Use of 3D printed models in medical education: a randomized control trial comparing 3D prints versus cadaveric materials for learning external cardiac anatomy. Anat Sci Educ 2016;9:213-21.

9. Lau IWW, Sun Z. Dimensional accuracy and clinical value of 3D printed models in congenital heart disease: a systematic review and meta-analysis. J Clin Med 2019;8:1483.
10. Loke YH, Harahsheh AS, Krieger A, Olivieri LJ. Usage of 3D models of tetralogy of Fallot for medical education: impact on learning congenital heart disease. BMC Med Educ 2017;17:54.

11. Wu AM, Wang K, Wang JS, Chen CH, Yang XD, Ni WF, et al. The addition of 3D printed models to enhance the teaching and learning of bone spatial anatomy and fractures for undergraduate students: a randomized controlled study. Ann Transl Med 2018;6:403.

12. Chen C, Cai L, Zheng W, Wang J, Guo X, Chen H. The efficacy of using 3D printing models in the treatment of fractures: a randomised clinical trial. BMC Muscoskel Disord 2019;20:65.

13. Savarese RG, Capobianco JD, Cox JJ. OMT review: a comprehensive review in osteopathic medicine, 3rd ed. Jacksonville, Florida: Legis Press; 2003.

14. Pujol S, Baldwin M, Nassiri J, Kikinis R, Shaffer K. Using 3D modeling techniques to enhance teaching of difficult anatomical concepts. Acad Radiol 2016;23:507-16.

15. Kuklovska Elizaveta DP. Amazon. Published 2018 Nov 6. Seattle, Washington. Available from: https://www.amazon.com/dp/ B07SZLZG5G/ref=cm_sw_em_r_mt_dp_pU4jFb115ZDZC [Accessed Nov 2018].

16. Gerefalk C, Rich A. Thingiverse website. Locking ball and socket arm - print and assembly optimized by KriLL3. Published 2016 Mar 16. Available from: https://www.thingiverse.com/thing: 1420341 [Accessed Nov 2018].

17. Mitsuhashi N, Fujieda K, Tamura T, Kawamoto S, Takagi T, Okubo K. BodyParts3D: 3D structure database for anatomical concepts. Nucleic Acids Res 2009;37:D782-5.

18. Lakens D. Calculating and reporting effect sizes to facilitate cumulative science: a practical primer for t-tests and ANOVAs. Front Psychol 2013;4:863.

19. Aimar A, Palermo A, Innocenti B. The role of 3D printing in medical applications: a state of the art. J Healthc Eng 2019;2019:1-10.

20. 3B Scientific. Classic flexible spine anatomy model with ribs and femur heads. Anatomy Warehouse. Available from: https:// anatomywarehouse.com/classic-flexible-spine-anatomy-modelwith-ribs-and-femur-heads-a-100148?gclid=Cj0KCQjwwOz6BRC gARIsAKEG4FUY94wye6VvjLBbcEZ_Z-AVoV9SOT_VwAvY7 DhWzQQESO1j0-FAdfoaAtj3EALw_wcB [Accessed Nov 2018]. 